\title{
Thermal conductivity of donor-doped GaN measured with $3 \omega$ and stationary methods
}

\author{
O. Churiukova, A. Jeżowski, P. Stachowiak, J. Mucha, and Z. Litwicki
}

W. Trzebiatowski Institute of Low Temperature and Structure Research Polish Academy of Sciences

2 Okólna Str., Wroclaw 50-422, Poland

E-mail: o.churiukova@int.pan.wroc.pl

P. Perlin and T. Suski

Institute of High Pressure Physics, Polish Academy of Sciences, 98 Al. Prymas Tysiaclecia, Warsaw 01-424, Poland

Received January 13, 2015, published online May 25, 2015

\begin{abstract}
The thermal conductivity of three single crystal samples of $n$-type gallium nitride with electron densities of $4.0 \cdot 10^{16}, 2.6 \cdot 10^{18}$, and $1.1 \cdot 10^{20} \mathrm{~cm}^{-3}$ has been determined in the temperature range 4-320 $\mathrm{K}$. The measurements were carried out within the $a b$ plane using the stationary method. The thermal conductivity depends strongly on the donor concentration. The analysis within the Callaway approach and the Debye model shows a significant influence of phonon-electron scattering on the thermal conductivity of the samples. In addition, some preliminary results obtained along the $c$ axes of GaN layered samples are presented. The latter measurements have been carried out using the $3 \omega$ method.
\end{abstract}

PACS: 44.10.+i Heat conduction;

63.20.kd Phonon-electron interactions;

68.60.Dv Thermal stability; thermal effects.

Keywords: thermal conductivity, GaN, thin film, phonon-electron scattering.

\section{Introduction}

In the last decade, GaN has attracted attention as a suitable material for various optoelectronic devices over the entire visible spectrum up to ultraviolet. It became a material to be used in high-power, high-frequency, high-temperature applications such as high-frequency transistors, laser diodes, microwave power sources, solar cells, blue and green LEDs or light emitters, and other lighting devices.

The performance and reliability of the majority of those devices rely critically on the efficiency of heat removal from the device-active region [1,2]. Therefore the thermal conductivity of $\mathrm{GaN}$ is of interest as a key parameter for designing high-power devices in which gallium nitride crystal is an important component.

Similarly, gallium nitride thin films are essential constituents of various functional devices in micro/nanoelectronics, optoelectronics, and micro-electro-mechanical systems $[3,4]$. And again, to achieve optimal thermal management and high reliability of the devices, the thermal characterization of these films and therefore fundamental understanding of the thermal properties of various $\mathrm{GaN}$ thin films is required.
While the thermal conductivity of a bulk crystal is easy to determine, the measurements of thermal conductivity of a thin film structure are much more complicated. Few experimental techniques allows investigation of thermal conductivity of layers a few hundred nanometers thick. One of them, the so called $3 \omega$ method, was developed by David G. Cahill [5].

\section{Experimental details}

There are several methods for growing gallium nitride crystals. Our samples were prepared using the ammonothermal technique. In this method the GaN feedstock is dissolved in a supercritical ammonia solution in one zone of a high pressure autoclave, then transported through the solution by convection, and, finally, crystallized in another zone, preferably on GaN seeds. The typical temperatures and pressures were respectively $500-900{ }^{\circ} \mathrm{C}$ and $0.1-0.3 \mathrm{GPa}[6]$.

In our experiment the temperature dependence of the thermal conductivity of GaN samples was determined by both the steady-state heat-flow and $3 \omega$ methods. We present our results for both bulk and layered samples. The 
bulk samples were single crystals $0.2 \mathrm{~mm}$ thick, $2 \mathrm{~mm}$ wide, and $10 \mathrm{~mm}$ high. They were studied with the steadyheat-flow method over the temperature range 5 to $320 \mathrm{~K}$. It should be emphasized that owing to the inherent overall instability of the thermal conductivity in our samples and its dependence on the thermal treatment, all measurements were carried out according to the same procedure: Prior to every thermal conductivity experiment the sample was kept for a couple of weeks at room temperature. Then, the sample was placed in the cryostat measuring chamber and then rapidly cooled down to liquid helium temperature, at which the measurements started. During measurements the temperature of the sample was varied only in one direction (heating) in order to obtain a set of data points from the lowest temperature to the highest. This procedure was the only way we could find to get a reproducible set of data without possible effects of the thermal history of the sample.

We also studied the thermal conductivity of layered GaN samples. These samples were prepared on a $1 \mathrm{~mm}$ thick sapphire plate. Every sample consisted of two layers. The lower one was a $450 \mathrm{~nm}$ layer of GaN:Si deposited directly on sapphire and the GaN:Al upper $450 \mathrm{~nm}$ thick layer was grown on the surface of the lower another. To determine the thermal conductivity of such films, the $3 \omega$ method [4] was utilized. For this method a special sample preparation technique is required. The samples must have a smooth flat surface, on which a special golden pattern (usually lithography deposited) is applied as shown in Fig. 1. During thermal conductivity measurements the golden pattern serves both as a heater and as a thermometer driven by an AC signal of frequency $\omega$. The metal line is warmed by Joule heating at frequency $2 \omega$. The temperature oscillations of the metal strip

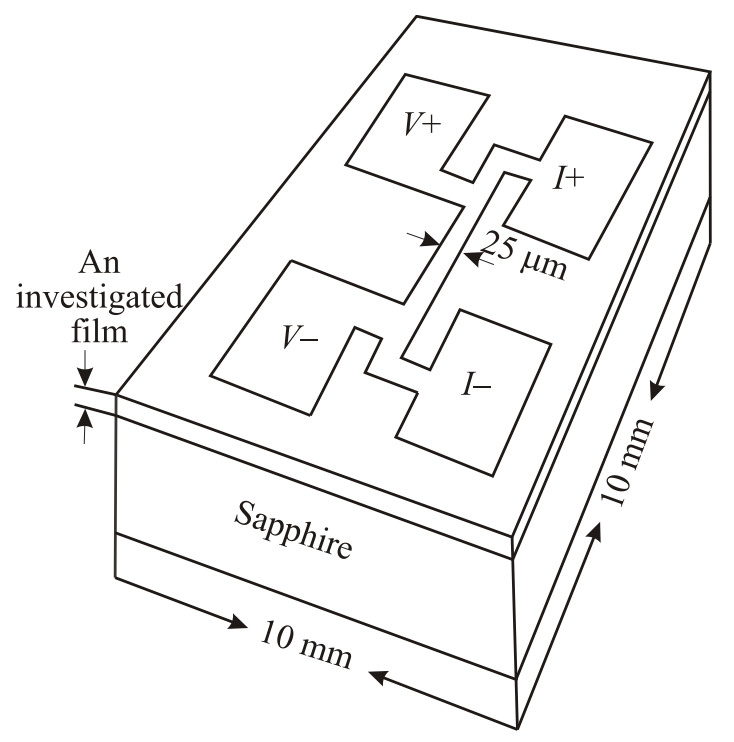

Fig. 1. Geometry of the golden pattern prepared on the surface of a thin film for determination of the thermal conductivity of the film by $3 \omega$ method. are measured through the small voltage oscillations at the third harmonic $3 \omega$ produced as a result of the temperaturedependent resistance of the metal strip.

A detailed geometry of a typical golden pattern grown on a thin-layer sample is shown in Fig. 1. We chose the necessary dimensions so that the width of the metal strip was substantially larger than the thickness of the layer under investigation. Therefore, the heat flow through the layer in question was one-dimensional. The heat passes through the sample uniformly across the width of the metal strip and the measured average surface temperature, without disturbing the heat flux. The sample prepared in this way was placed on the measurement table inside the cryostat. The temperature of the table was monitored by a precise temperature controller; the measurements were carried out over the temperature range $50-300 \mathrm{~K}$.

\section{Results and discussion}

The results of our thermal conductivity versus temperature measurements obtained with the stationary method for three bulk GaN samples with different free electron concentration $10^{16}(\mathrm{~S} 1), 10^{18}(\mathrm{~S} 2)$ and $10^{20}(\mathrm{~S} 3)$ per $\mathrm{cm}^{3}$ shown in Fig. 2. The thermal conductivity depends strongly on the donor concentration. The thermal conductivities at maximum amount to 1200,200 and $100 \mathrm{~W} \cdot \mathrm{m}^{-1} \cdot \mathrm{K}^{-1}$ while at room temperature they were 280,135 and $70 \mathrm{~W} \cdot \mathrm{m}^{-1} \cdot \mathrm{K}^{-1}$ for samples S1, S2, and S3, respectively.

In the simplest case, at the lowermost temperatures the thermal conductivity $\kappa$ of a dielectric crystal depends on its linear dimensions since the phonon scattering processes are dominated by scattering by the crystal (or its grains) boundaries. This results in the low-temperature $\kappa \propto T^{3}$ dependence. In the intermediate temperature range the phonon scattering becomes dominated by three-phonon $U$-processes, which results in a characteristic exponential decrease of the thermal conductivity with increasing tem-

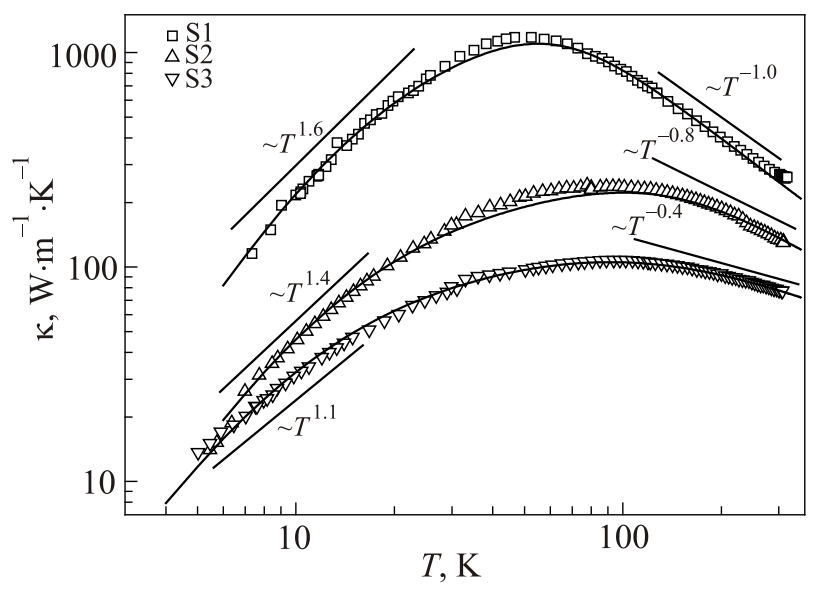

Fig. 2. Thermal conductivity of GaN single crystals with different donor concentration. The solid curves are plots of the best fit of Eq. (1) to the data obtained in the thermal conductivity experiment. 
perature. At still higher temperatures the exponential dependence gradually becomes weaker to finally follow the Leibfried-Schlömann law [7], $\kappa \propto 1 / T$. In case of a real crystal this temperature dependence of the thermal conductivity is modified significantly by interactions of phonon with crystal structure imperfections other than boundaries. The modifications are usually better pronounced at low temperatures. The departure of the thermal conductivity reported here for GaN crystals from the picture sketched above testifies to presence of more complicated phonon scattering mechanisms beside boundary scattering and three-phonon processes. To assess the significance of any phonon scattering mechanism for the thermal conductivity of our gallium nitride crystals the Callaway analysis [8] was done.

In the Callaway method each phonon scattering mechanism is represented by its characteristic relaxation time, which is a function of the phonon frequency. In this method, within the frame of the Debye model, the dependence of the phonon thermal conductivity $\kappa_{\mathrm{ph}}$ of a crystal on temperature $\mathrm{T}$ can be written as

$$
\kappa_{\mathrm{ph}}=\kappa_{1}+\kappa_{2},
$$

where

$$
\kappa_{1}=\frac{k_{B}}{2 \pi^{2} v}\left(\frac{k_{B}}{\hbar}\right)^{3} T^{3} \int_{0}^{\frac{\theta_{D}}{T}} \frac{\tau_{C} x^{4} \mathrm{e}^{x}}{\left(\mathrm{e}^{x}-1\right)^{2}} d x,
$$

and

$$
\kappa_{2}=\frac{k_{B}}{2 \pi^{2} v}\left(\frac{k_{B}}{\hbar}\right)^{3} T^{3} \frac{\left[\int_{0}^{\frac{\theta_{D}}{T}}\left(\frac{\tau_{C}}{\tau_{R}}\right) x^{4} \mathrm{e}^{x}\left(\mathrm{e}^{x}-1\right)^{-2} d x\right]^{2}}{\int_{0}^{\frac{\theta_{D}}{T}}\left(\frac{\tau_{C}}{\tau_{R} \tau_{N}}\right) x^{4} \mathrm{e}^{x}\left(\mathrm{e}^{x}-1\right)^{-2} d x},
$$

where $\tau_{i}$ are the relaxation times of phonon scattering,

$$
G=\frac{k_{B}^{4}}{2 \pi^{2} v \hbar^{3}}, \quad x=\frac{\hbar \omega}{k_{B} T}
$$

and $v$ is the effective velocity of phonons.

Assuming that different scattering processes do not influence each other, the resulting inverse phonon scattering time $\tau_{C}^{-1}$ (phonon scattering rate) can be written as

$$
\tau_{C}^{-1}=\tau_{R}^{-1}+\tau_{N}^{-1}
$$

where

$$
\tau_{R}^{-1}=\tau_{B}^{-1}+\tau_{P}^{-1}+\tau_{D}^{-1}+\tau_{U}^{-1}
$$

The subscripts $B, P, D, U$ denote the relaxation times for scattering of phonons by grain boundaries, point defects (Rayleigh scattering), electrons, and $U$-processes, respectively. The Callaway method consists in a simultaneous variation of the relaxation time parameters involved for different phonon scattering mechanisms in order to obtain the best possible fit of the expression in Eq. (1) to the experimental data.

The analysis of our results depicted in Fig. 2 using the Callaway method clearly show that among all phonon scattering mechanisms taken into account the phonon-electron scattering is the most important at low temperatures. Therefore this mechanism determines the low- $T$ thermal conductivity of gallium nitride bulk crystals as a function of temperature. The high probability of these processes also explains the changes of the thermal conductivity with increasing donor concentration.

The results of our measurements carried out with the $3 \omega$ method for layered GaN samples are shown in Figs. 3 and 4 . Figure 4 shows the final results calculated from direct experimental data, an example of which is depicted in Fig. 3. As mentioned above, the electrical current warms up the specially prepared golden heater at a frequency of $2 \omega$ due to the Joule heat, producing temperature oscillations also at a frequency of $2 \omega$ with the amplitude $\Delta T_{2 \omega}$. Since the resistance of the metal heater increases linearly with temperature, the temperature oscillations result in the resistance oscillations in the metal strip at a frequency of $3 \omega$. Thus, the change of temperature of the metal strip can be obtained from the measurements of the third harmonic of the voltage, $U_{3 \omega}$. The amplitude of the temperature oscillations can be calculated from the equation

$$
\Delta T_{3 \omega}=\frac{2 U_{3 \omega}}{I_{0} \alpha},
$$

where $I_{0}$ is the rms value of the electric current along the golden strip, $\alpha$ is the temperature coefficient of the golden heater resistivity $R\left(\alpha=\frac{d R}{d T}\right)$. Since the metal heater is in a very good thermal contact with the underlying substrate,



Fig. 3. Example of the temperature oscillation amplitude on the surface of a GaN layer. 


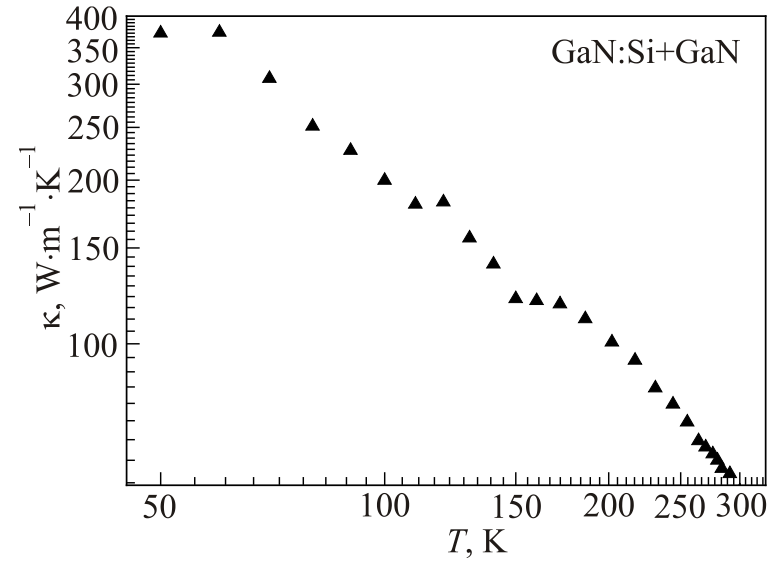

Fig. 4. Combined thermal conductivity of the GaN layers and the substrate.

the amplitude of the temperature oscillations depends not only on $I_{0}$ and $\alpha$, but also on the thermal conductivity of the substrate and the frequency of the applied signal. Mathematical considerations of the phenomenon [9] lead to the equation:

$$
\kappa=\frac{\beta}{R} \cdot \frac{U_{1 \omega}^{3}}{4 \pi l} \cdot \frac{\ln \left(f_{2} / f_{1}\right)}{U_{3 \omega_{f 1}}-U_{3 \omega_{f 2}}},
$$

where $U_{3 \omega_{f 1}}$ and $U_{3 \omega_{f 2}}$ are the third harmonic voltage at input current frequency of $f_{1}$ and $f_{2}$, respectively, $l$ is the length of the metal strip, $\beta=\frac{\alpha}{R}$. Therefore, from any direct experimental dependence such as the one shown in Fig. 3 one can obtain a single value of the thermal conductivity of the substrate at a given temperature. A set of dependencies for different temperatures allows us to calculate the curve such as the one shown in Fig. 4. It should be stressed here that the dependence in Fig. 4 shows a sum of the thermal conductivities of the GaN layers and the sapphire substrate. To separate the thermal conductivity of the layers themselves, a special experiment on the sapphire substrate alone is necessary.

\section{Conclusions}

The temperature dependence of the thermal conductivity of three single crystal GaN samples containing different concentrations of electrons in the conduction band was determined over the temperature range 5-300 K. The experimental results were analyzed in the phonon relaxation time approximation using the Callaway method. We have also measured the thermal conductivity of thin films of GaN deposited on sapphire using the $3 \omega$ method in the temperature range from 50 to $320 \mathrm{~K}$.

\section{Acknowledgements}

The work was supported by Wroclaw Research Centre EIT+ within the project "The Application of Nanotechnology in Advanced Materials” — NanoMat (POIG.01.01.0202-002/08) co-financed by the European Regional Development Fund (operational Programme Innovative Economy, 1.1.2).

1. M. Kamano, M. Haraguchi, T. Niwaki, M. Fukui, M. Kuwahara, T. Okamoto, and T. Mukai, Jpn. J. Appl. Phys. 41, 5034 (2002).

2. G.A. Slack, L.J. Schowalter, D. Morelli, and J.A. Freitas, Jr., J. Cryst. Growth 246, 287 (2002).

3. Z.-X. Zong, Z.-J. Qiu, S.-L. Zhang, R. Streiter, and R. Liu, J. Appl. Phys. 109, 063502 (2011).

4. S.-M. Lee and D.G. Cahill, J. Appl. Phys. 81, 2590 (1997).

5. D.G. Cahill, Rev. Sci. Instrum. 61, 802 (1990).

6. R. Kucharski, M. Rudziński, M. Zając, R. Doradziński, J. Garczyński, L. Sierzputowski, R. Kudrawiec, J. Serafińczuk, W. Strupiński, and R. Dwiliński, Appl. Phys. Lett. 95, 131119 (2009).

7. G. Leibfried and E. Schlömann, Nach. Akad. Wiss. Göttingen, Math. Phys. Klasse 4, 71 (1954).

8. J. Callaway, Phys. Rev. 13, 4 (1959).

9. F. Faghani, Thermal Conductivity Measurement of PEDOT:PSS by 3-omega Technique, Linköping Universitet Tekniska Högskolan (2010). 